\title{
Receipt of General Medical Care by Colorectal Cancer Patients: A Longitudinal Study
}

\author{
Laura-Mae Baldwin, MD, MPH, Sharon A. Dobie, MCP, MD, Yong Cai, PhD, \\ Barry G. Saver, MD, MPH, Pamela K. Green, MPH, PhD, and C. Y. Wang, PhD
}

Background: Cancer diagnosis has the potential to overshadow patients' general medical care needs. This study examined changes in general medical care among elderly patients with colorectal cancer (CRC), from before diagnosis through long-term survival.

Methods: This longitudinal cohort study used 1993 to 1999 Surveillance, Epidemiology, and End Results and 1991 to 2001 Medicare claims data for 22,161 patients with stage 0 to 3 CRC and 81,669 controls aged 67 to 89 years. Outcomes were preventive services (influenza vaccination, mammography) and, among diabetics, HgbA1c and lipid testing in the phase before diagnosis, the phase after initial treatment, the surveillance phase, and the survival care phase. Logistic regression provided adjusted relative risks of care receipt for patients with stage 0 to 1 cancer, stage 2 to 3 cancer, and no cancer.

Results: In the phase before diagnosis through the surveillance phase, patients with stage 0 to 1 CRC had the highest annual preventive service rates. Patients with stage 2 to 3 CRC made substantial gains in preventive service use, especially mammography, after diagnosis (influenza vaccination, $46.4 \%$ before diagnosis to $50.2 \%$ after initial treatment; mammography, $31.4 \%$ before diagnosis to $40.2 \%$ after initial treatment) but not in diabetes care (eg, HgbA1c, $53.4 \%$ before diagnosis to $54.9 \%$ after initial treatment).

Conclusions: CRC diagnosis seems to facilitate receipt of preventive services but not diabetes care for elderly, later-stage patients. Additional strategies such as strengthening partnerships between cancer patients, primary care physicians, and cancer care physicians are needed to improve care for a chronic disease like diabetes. (J Am Board Fam Med 2011;24:57-68.)

Keywords: Cancer, Colorectal Cancer, Medicare, Quality of Care, Survivorship

Approximately $70 \%$ of all cancer patients are aged $\geq 65$ years and enter the cancer care system with a broad range of medical conditions and preventive care needs. ${ }^{1-4}$ However, cancer diagnosis has the potential to overshadow these general medical care

This article was externally peer reviewed.

Submitted 31 March 2010; revised 11 October 2010; accepted 15 October 2010.

From the Department of Family Medicine, University of Washington, Seattle (L-MB, SAD, PKG); the Department of Sociology, University of North Carolina-Chapel Hill, Chapel Hill (YC); the Department of Family Medicine and Community Health, University of Massachusetts, Worcester (BGS); and the Division of Public Health Sciences, Fred Hutchinson Cancer Research Center, Seattle, WA (CYW).

Funding: Funding was provided by the National Cancer Institute (1 R01 CA104935).

Conflict of interest: none declared.

Corresponding author: Laura-Mae Baldwin, MD, MPH, Department of Family Medicine, University of Washington, Box 354982, Seattle, WA 98195-4982 (E-mail: lmb@u. washington.edu). needs. Several studies suggest that addressing cancer patients' general medical care can have an important impact on survival. ${ }^{5-10}$ Higher mortality among black patients who are receiving cancer treatment comparable to that being received by white patients is largely related to deaths from other conditions. ${ }^{5}$ Influenza vaccination receipt by patients with advanced-stage colorectal cancer (CRC) has been associated with increased survival. ${ }^{6}$ Beyond survival, Ramsey et al ${ }^{11}$ have demonstrated that noncancer comorbidity is the strongest predictor of quality of life for most CRC survivors.

Relatively little research has been published about general medical care among cancer patients. Earle and Neville ${ }^{12}$ found that 5 -year CRC survivors received less preventive and chronic disease care than noncancer patients matched on demographics and geography. Lower influenza vaccination rates have been found among elderly patients 
with advanced-stage CRC in chemotherapy compared with their elderly noncancer counterparts. ${ }^{6}$ Conversely, Snyder et $\mathrm{al}^{13}$ found increases in influenza vaccination but decreases in mammography and cervical cancer screening in the 5 years after initial treatment among patients with stage 1 to 3 CRC. However, none of these studies examined whether there was a change in these patients' receipt of general medical care services before and after cancer diagnosis, nor did they compare cancer patients' general medical care service receipt to that of a control population.

This study builds on prior research by examining receipt of preventive and diabetes care among elderly cancer and noncancer patients from the year before diagnosis through 3 cancer care phases: after initial treatment, surveillance, and long-term survival. We chose diabetes because it is a common chronic medical condition with growing prevalence and recommended diabetes care includes testing measurable by claims data. We hypothesized that cancer diagnosis would be associated with a decline in receipt of preventive and diabetes care that recovered as the time from diagnosis lengthened. This research specifically addresses the Institute of Medicine's call for increased attention to the quality of care for cancer survivors. ${ }^{14}$

\section{Methods}

Data Sources

This study used the Surveillance, Epidemiology, and End Results (SEER) cancer registry data linked with Medicare claims to identify cancer patients, and Medicare claims from the annual 5\% random sample of Medicare beneficiaries without cancer who resided in SEER registry areas to identify controls. ${ }^{15}$ SEER data include patient demographics and cancer type and stage. Medicare data include enrollment dates, health maintenance organization membership, patient demographics and, for fee-for-service beneficiaries, new billed claims that include diagnoses, tests, and procedures provided in hospitals, physician offices, and clinics. The American Medical Association's (AMA) Physician Masterfile data linked to the Medicare claims provided physician specialty.

\section{Study Population and Care Phases}

Cancer patients had CRC in stages 0 to 3 reported between 1993 and 1999 and were 67 to 89 years old at diagnosis $(\mathrm{n}=64,410)$. Sequentially excluded were individuals missing diagnosis month $(\mathrm{n}=$ 114), those with a simultaneous stage 4 cancer $(\mathrm{n}=$ $244)$, those with atypical histology $(\mathrm{n}=284)$, and those diagnosed at autopsy $(\mathrm{n}=108)$. Noncancer controls, made up of the complete $5 \%$ random sample of all Medicare beneficiaries who lived in SEER registry areas between 1993 and 1999, were randomly assigned a pseudodiagnosis (from here on called "diagnosis") month and year between January 1, 1993, and December 31, 1999, so that, like cancer patients, they had an anchor date from which to measure study variables and care phases. The 161,115 noncancer controls who were 67 to 89 years old at the assigned diagnosis date were included. Because we wanted to examine the association between a primary CRC diagnosis and receipt of general medical care, and because additional cancer diagnoses might influence receipt of general medical care, we excluded patients with additional SEER cancer diagnoses through 1999 (n $=13,989)$.

We defined 5 care phases for the study (Figure 1): before diagnosis (13 months before 1 month prior to the diagnosis month); washout (one month before through the fifth month after the diagnosis month); after initial treatment (the year after washout); surveillance ( 2 to 4 years after washout); and survival ( 5 to 7 years after washout). We also gathered data in the year before the phase before diag-

Figure 1. Study care phases.

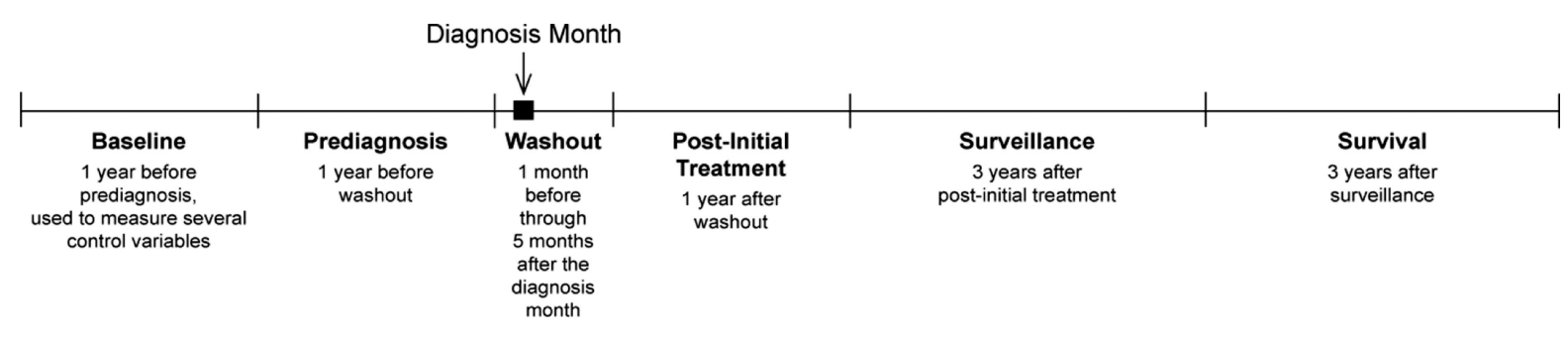


nosis to define pre-existing comorbidity, the number of hospitalizations, and diabetes diagnosis. We required continuous enrollment in fee-for-service parts A and B Medicare from the year before the phase before diagnosis through the phase after initial treatment (resulting in exclusion of 27,510 cases and 79,446 controls). Based on SEER/ Medicare data availability, cases $(n=22,161)$ and controls $(\mathrm{n}=81,669)$ with diagnoses starting in February 1993 were included.

Study individuals contributed at least 2 data years (before diagnosis and after initial treatment) and up to 8 data years (Table 1). Observation years with evidence of metastatic disease (eg, ablation of liver lesion; see Appendix for codes) or recurrence treatment (ie, chemotherapy and radiation administration; see Appendix for codes) were censored, as were all subsequent years, because we surmised that recurrence or metastasis would decrease both screening and monitoring of chronic disease. The average number of study years varied significantly by cancer diagnosis and stage: 4.7 for controls, 4.4 for patients with stage 0 to $1 \mathrm{CRC}$, and 4.0 for patients with stage 2 to 3 CRC.

\section{Outcome Variables}

We measured 2 types of noncancer care: (1) preventive care (see Appendix for codes), specifically mammography for women ages 67 to 74 years $(\mathrm{n}=$ 23,982 ) and influenza vaccination; and (2) diabetes care as an example of chronic disease care, evaluating glycosylated hemoglobin (HgbA1c) and lipid test measurements (see Appendix for codes) among diabetics $(\mathrm{n}=11,858)$. These measures were standard, guideline-recommended care for older adults at the time of this study. ${ }^{16,17}$ Individuals with diabetes had one inpatient or 2 outpatient International Classification of Diseases, Ninth Revision, Clinical Modification, diagnosis codes indicating diabetes in the year before prediagnosis (250.XX). Because influenza vaccination was not a Medicarecovered service until May 1993, we allowed a 7-month ramp-up and included only individuals with a phase before diagnosis year starting in January $1994(\mathrm{n}=73,421)$ in those analyses.

\section{Independent Variable of Interest}

Our independent variable of interest was CRC diagnosis status. Initial analyses identified substantial differences in noncancer care between early-stage (stages 0 and 1) and later-stage (stages 2 and 3 ) cancers, so we separated these groups.

\section{Covariates}

Time-independent covariates included patient sex; race (white, black, Hispanic, Asian, or other); ZIP code-based median annual household income in the diagnosis year, ZIP code-based percent of the population $\geq 25$ years old who were high school graduates in the diagnosis year; and reason for initial Medicare entitlement (disability or end-stage renal disease vs age $\geq 65$ ). The 2000 census provided the ZIP code-based variables. Time-dependent variables included age at the observation year's start (67 to 69 years, 70 to 74 years, 75 to 79 years, and 80 to 89 years); the number of hospitalizations

Table 1. Number of Persons in Each Study Observation Year by Study Group

\begin{tabular}{lcccc}
\hline & & \multicolumn{2}{c}{ CRC Cases } \\
\cline { 3 - 4 } & Total & Stage 0-1 & Stage 2-3 & Controls \\
\hline Year before prediagnosis phase (year -2) & 103,830 & 8,865 & 13,296 & 81,669 \\
Phase before diagnosis (year -1) & 103,830 & 8,865 & 13,296 & 81,669 \\
Phase after initial treatment (year 1) & 103,830 & 8,865 & 13,296 & 81,669 \\
Surveillance phase & & & & 70,679 \\
$\quad$ Year 2 & 88,859 & 7,548 & 7,672 & 54,714 \\
Year 3 & 67,959 & 5,573 & 5,366 & 40,711 \\
$\quad$ Year 4 & 50,159 & 4,082 & 3,575 & 28,734 \\
Survival phase & & & 2,240 & 18,604 \\
$\quad$ Year 5 & 35,134 & 2,825 & 1,140 & 10,131 \\
$\quad$ Year 6 & 22,635 & 1,791 & 991 & \\
$\quad$ Year 7 & 12,262 & & & 700 \\
\hline
\end{tabular}

CRC, colorectal cancer. 
during the year before observation $(0,1,2,3,4$, $\geq 5$ ); the number of visits to noncancer care physicians during the observation year; the number of visits to cancer care physicians (general or colorectal surgeon, medical oncologist, radiation oncologist) during the observation year; and comorbidity during the year before observation (using the $\mathrm{Na}$ tional Cancer Institute's combined comorbidity index specific to CRC patients ${ }^{18}$ ). We identified cancer and noncancer care physicians using linked data about primary specialty, secondary specialty, and board certification from the 1993, 1997, and 2002 AMA Physician Masterfiles. When AMA Physician Masterfile data were missing, we used the specialty code from the Medicare claims file.

\section{Analysis}

We compared the characteristics of the 3 study groups from which our study samples were drawn (patients with stage 0 to $1 \mathrm{CRC}$, patients with stage 2 to 3 CRC cases, and controls) in the phase before diagnosis. We calculated unadjusted annual rates of receipt of preventive and diabetes services by group in each phase before diagnosis, after initial treatment, surveillance, and survival phase year; we then tested for trends in receipt of these services within each of the 3 study groups over the 8 observation years and for differences in trends between our 3 study groups using the general estimating equation (GEE) approach with a logit link, using year, study group, and their interaction terms in the models. We used GEE with an unstructured correlation matrix to account for clustering by individual because each could contribute up to 8 data years. We excluded the washout period in our analyses to account for the likelihood that noncancer care would be logically deferred because of pressing cancer diagnosis and treatment needs during the time period immediately before and after cancer diagnosis.

Next, we conducted GEE analysis (logit link, unstructured correlation matrix) with cases or controls in different care phases (eg, stage 0 to 1 CRC cases in the phase before diagnosis, stage 2 to 3 CRC cases in the phase after initial treatment) as the independent variables and receipt of preventive and diabetes services in each observation year as outcomes. We adjusted for patient age and sex (when applicable) in all the models and included all other covariates described above if they were significant predictors of our outcomes at the $P \leq .05$ level. The diabetes care models also adjusted for the presence of a diabetes diagnosis (one inpatient or 2 outpatient International Classification of Diseases, Ninth Revision, Clinical Modification diagnosis codes) during the year before the observation year to account for variation in diabetes disease activity among individuals. Adjusted odds ratios were converted to relative risks using published methods. ${ }^{19}$ We used SAS 9.2 software (SAS Institute, Inc., Cary, NC) to conduct all analyses.

\section{Results \\ Study Group Characteristics}

Controls were the youngest of the 3 study groups and patients with stage 2 to 3 CRC were the oldest (Table 2). The majority of all 3 groups were women, although controls were most likely $(62.8 \%)$ and patients with stage 0 to 1 CRC least likely $(53.9 \%)$ to be women. Before diagnosis, controls had the least comorbidity $(76.1 \%$; comorbidity score, $\leq 0)$ and patients with stage 0 to $1 \mathrm{CRC}$ had the most comorbidity $(71.7 \%$; comorbidity score, $\leq 0)$. Before diagnosis, patients with stage 0 to $1 \mathrm{CRC}$ had the greatest number of visits to noncancer care physicians. These findings were generally consistent across the influenza, mammography, and diabetes study subsamples, though the mammography subsample included only women, and the comorbidity index was not significantly different across groups in the diabetes subsample.

\section{Noncancer Care Receipt}

\section{Influenza Vaccination}

Before diagnosis, patients with stage 0 to 1 CRC had significantly higher annual influenza vaccination rates (unadjusted annual rates with confidence intervals (CI), $50.9 \%$ [ $49.6 \%$ to $52.1 \%]$ ]) than both patients with stage 2 to 3 CRC (unadjusted annual rates with CI, 46.4\% [45.4\%, 47.4\%]) and controls (unadjusted annual rates with CI, 46.1\% [45.7\%$46.5 \%]$ ) in unadjusted analysis (Table 3); they also had higher likelihood of influenza vaccination in adjusted analyses (adjusted relative risk [aRR], 1.09; 95\% CI, 1.06-1.12); aRR, 0.99; 95\% CI, 0.971.01; aRR, 1.0 [reference group], respectively) (Figure 2). In adjusted analysis, patients with stage 0 to 1 CRC had a higher likelihood of influenza vaccination than controls throughout the study phases and had a higher likelihood of influenza vaccination 


\begin{tabular}{|c|c|c|c|}
\hline & \multicolumn{2}{|c|}{ CRC Cases } & \multirow[b]{2}{*}{$\begin{array}{c}\text { Controls } \\
(\mathrm{n}=81,669)\end{array}$} \\
\hline & $\begin{array}{l}\text { Stage } 0-1 \\
(\mathrm{n}=8,865)\end{array}$ & $\begin{array}{c}\text { Stage } 2-3 \\
(\mathrm{n}=13,296)\end{array}$ & \\
\hline \multicolumn{4}{|l|}{ Age, years* } \\
\hline $67-69$ & 15.6 & 13.8 & 19.5 \\
\hline $70-74$ & 29.4 & 27.5 & 32.3 \\
\hline $75-79$ & 28.0 & 27.5 & 25.1 \\
\hline $80-89$ & 27.1 & 31.2 & 23.1 \\
\hline Female* & 53.9 & 56.6 & 62.8 \\
\hline \multicolumn{4}{|l|}{ Race/ethnicity* } \\
\hline White & 86.6 & 87.2 & 84.8 \\
\hline Asian & 3.5 & 3.3 & 3.8 \\
\hline Black & 6.3 & 6.1 & 6.6 \\
\hline Hispanic & 1.0 & 1.1 & 2.2 \\
\hline Other & 2.6 & 2.3 & 2.7 \\
\hline \multicolumn{4}{|c|}{$\begin{array}{l}\text { National Cancer Institute combined comorbidity index during } \\
\text { the phase before diagnosis year* }\end{array}$} \\
\hline$\leq 0$ & 71.7 & 74.7 & 76.1 \\
\hline$>0-0.5$ & 14.6 & 13.5 & 12.4 \\
\hline$>0.5-1.0$ & 8.1 & 7.5 & 7.4 \\
\hline$>1.0$ & 5.6 & 4.4 & 4.1 \\
\hline \multicolumn{4}{|c|}{ Hospitalizations during the phase before diagnosis year ${ }^{\dagger}$} \\
\hline 0 & 85.3 & 86.9 & 86.6 \\
\hline 1 & 11.1 & 10.1 & 10.1 \\
\hline 2 & 2.6 & 2.1 & 2.4 \\
\hline 3 & 0.8 & 0.6 & 0.6 \\
\hline 4 & 0.2 & 0.3 & 0.2 \\
\hline$\geq 5$ & 0.1 & 0.1 & 0.1 \\
\hline \multicolumn{4}{|c|}{ Annual visits to cancer care physicians (mean $[\mathrm{SD}])^{\ddagger}$} \\
\hline Phase after initial treatment* & $1.4(3.2)$ & $4.6(7.1)$ & $0.3(1.4)$ \\
\hline Surveillance phase* & $0.7(1.7)$ & $1.3(2.1)$ & $0.3(1.4)$ \\
\hline Survival phase* & $0.5(1.5)$ & $0.8(1.6)$ & $0.4(1.5)$ \\
\hline \multicolumn{4}{|c|}{$\begin{array}{l}\text { Visits to noncancer care physicians during the phase before } \\
\text { diagnosis year* }\end{array}$} \\
\hline$\leq 7$ & 42.6 & 50.0 & 50.1 \\
\hline $8-15$ & 33.0 & 30.2 & 29.2 \\
\hline $16-25$ & 16.6 & 14.2 & 14.2 \\
\hline$>25$ & 7.8 & 5.6 & 6.5 \\
\hline \multicolumn{4}{|l|}{ Reason for Medicare eligibility $\$$} \\
\hline Age $\geq 65$ years & 94.1 & 94.7 & 93.9 \\
\hline Disability/end-stage renal disease & 5.9 & 5.3 & 6.1 \\
\hline \multicolumn{4}{|c|}{$\begin{array}{l}\text { Percent of those } \geq 25 \text { years old who graduated from high school } \\
\text { in ZIP code }\end{array}$} \\
\hline $0-50$ & 1.7 & 1.7 & 2.1 \\
\hline $50-75$ & 16.0 & 16.2 & 17.6 \\
\hline $75-90$ & 54.8 & 54.5 & 52.2 \\
\hline$>90$ & 27.6 & 27.7 & 28.1 \\
\hline \multicolumn{4}{|c|}{ Median annual household income in ZIP code $^{* \|}$} \\
\hline$\leq \$ 30,000$ & 8.2 & 8.6 & 10.3 \\
\hline$>\$ 30,000-45,000$ & 38.4 & 39.0 & 38.2 \\
\hline$>\$ 45,000$ & 53.4 & 52.4 & 51.5 \\
\hline
\end{tabular}

Values provided as \% unless otherwise specified.

${ }^{*} P \leq .001$.

${ }^{\dagger} P \leq .05$.

${ }^{\ddagger}$ Cancer care physicians include general surgeons, colorectal surgeons, medical oncologists, radiation oncologists.

${ }^{\S} P \leq .01$.

"Missing values: people $\geq 25$ years old who graduated from high school in ZIP code (\%): stage 0-1, n = 207 (2.3\%); stage 2-3, n = $300(2.3 \%)$; controls, $\mathrm{n}=2274(2.8 \%)$; median annual household income in ZIP code: stage $0-1, \mathrm{n}=210(2.4 \%)$; stage $2-3, \mathrm{n}=301$ (2.3\%); controls, $\mathrm{n}=2286(2.8 \%)$.

CRC. 


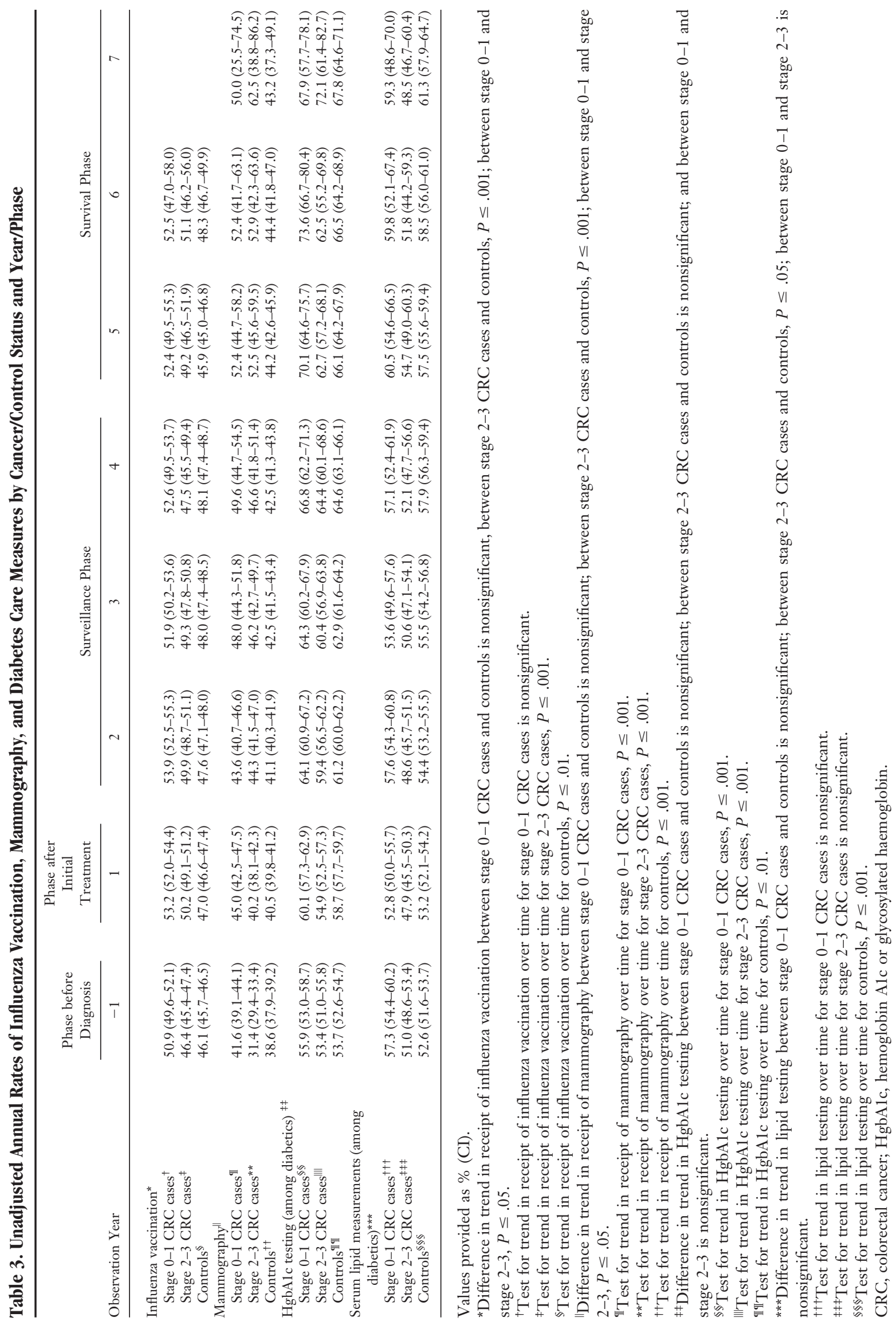


Figure 2. Adjusted relative risk of influenza vaccination, mammography, and diabetes care measures (hemoglobin A1c and lipid testing) by cancer/control status and phase. Adjusted for the following variables: age, sex (when applicable), race ethnicity, median annual household income in ZIP code percent of those $\geq 25$ years old who graduated from high school in zip code, reason for Medicare, National Cancer Institue combined comorbidity index, and number of hospitalizations. Note that relative risk scales differ for each measure.
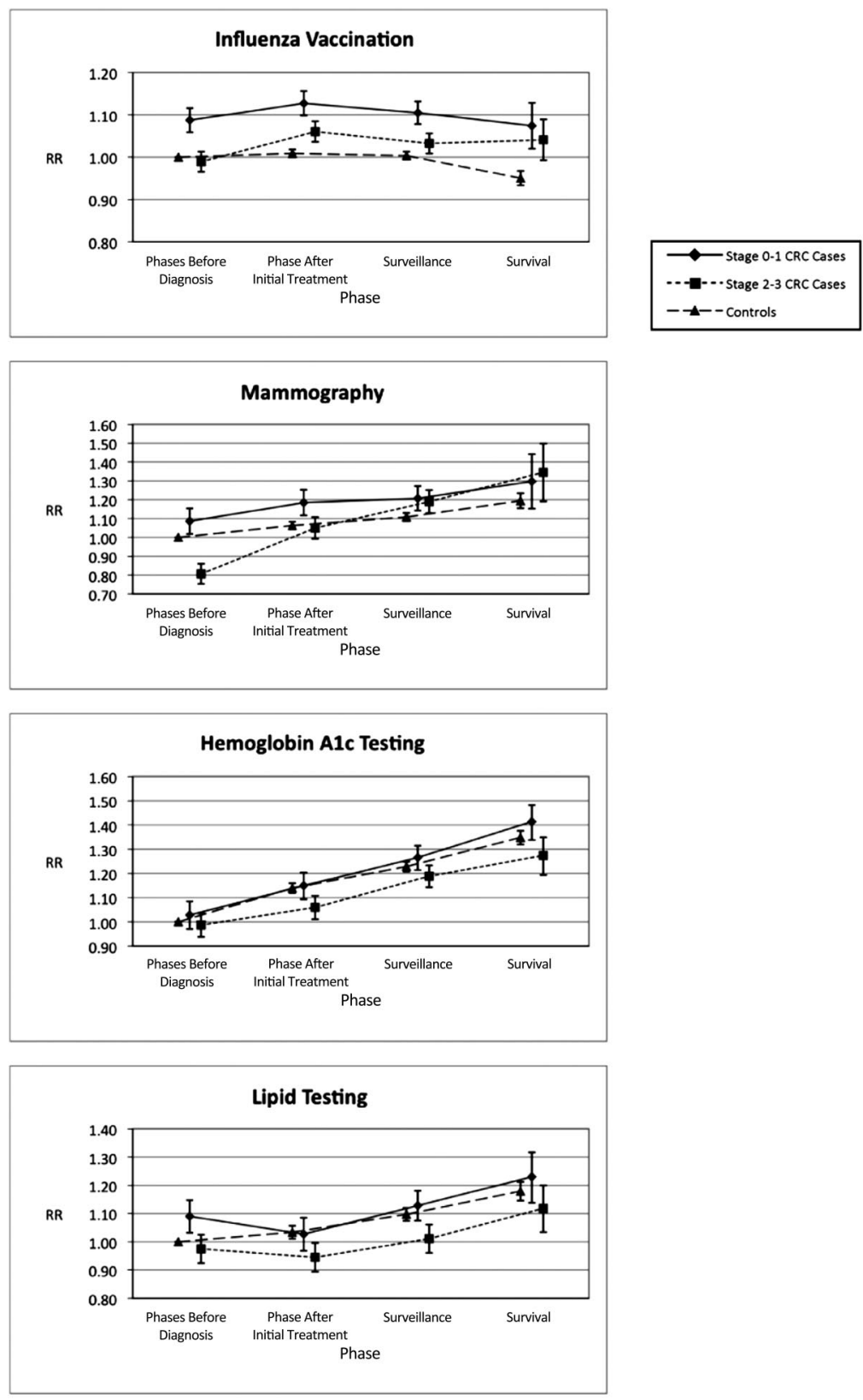

$\mathrm{RR}=$ relative risk

than patients with stage 2 to 3 CRC patients until the survival phase (Figure 2). Patients with stage 2 to 3 CRC significantly increased their likelihood of influenza vaccination between the phases before diagnosis and after initial treatment, and they largely sustained these increases (Figure 2). Visits with noncancer and cancer providers explained a substantial proportion of the higher likelihood of influenza vaccination among cancer patients after diagnosis, though during the survival phase significant differences in the likelihood of vaccination between both patients with stage 0 to $1 \mathrm{CRC}$ and those with stage 2 to 3 CRC and controls remained (results not shown). 


\section{Mammography}

Before diagnosis, patients with stage 0 to 1 CRC had the highest annual mammography rate $(41.6 \%$ [39.1\%-44.1\%]); patients with stage 2 to 3 CRC had a significantly lower annual mammography rate $(31.4 \%$ [29.4\%-33.4\%]) than both patients with stage 0 to 1 CRC $(41.6 \%$ [39.1\%-44.1\%]) and controls $(38.6 \%[37.9 \%-39.2 \%])$ in unadjusted analyses (Table 3). Patients with stage 2 to 3 CRC also had a lower likelihood of mammography (aRR, $0.81 ; 95 \%$ CI, 0.75-0.86) in adjusted analyses and did patients with stage 2 to 3 CRC (aRR, 1.09; 95\% CI, 1.02-1.15) and controls (aRR, 1.0 [reference group]) (Figure 2). All 3 study groups demonstrated significant increases in annual mammography rates over the observation years $(P \leq .001$ for trend tests); the group with stage 2 to 3 CRC had a greater increase in annual mammography rates than both the control $(P \leq .001)$ and stage 0 to 1 CRC $(P \leq .001)$ groups (Table 3$)$. In adjusted analysis (Figure 2), the likelihood of mammography among patients with stage 2 to $3 \mathrm{CRC}$ reached that of controls in the period after initial treatment and reached that of patients with stage 0 to $1 \mathrm{CRC}$ in the surveillance and survival phases. Adjusting for physician visits did not change these findings (results not shown).

\section{Diabetes Care}

Before diagnosis, annual HgbA1c rates from the unadjusted analysis were similar for the 3 study groups: stage 0 to $1 \mathrm{CRC}, 55.9 \%(53.0 \%-58.7 \%)$; stage 2 to 3 CRC, $53.4 \%(51.0 \%-55.8 \%)$; controls, $53.7 \%(52.6 \%-54.7 \%)$; however, annual lipid testing rates were higher among patients with stage 0 to 1 CRC (unadjusted annual rate, $57.3 \%$ [54.4\%$60.2 \%]$ ) compared with both controls (unadjusted annual rate, $52.6 \%[51.6 \%-53.7 \%])$ and patients with stage 2 to 3 CRC (unadjusted annual rate, $51.0 \%$ [48.6\%-53.4\%]) (Table 3). These findings did not change in adjusted analysis (Figure 2). In unadjusted analysis, the control group demonstrated increasing annual rates of HgbA1c and lipid testing over the observation period $(P \leq .001$; Table 3); in adjusted analysis, the likelihood of controls receiving HgbA1c and lipid testing increased significantly during each successive study phase (Figure 2). Like controls, the likelihood of HgbA1c testing among patients with stage 0 to 1 CRC significantly increased during each successive study phase in the adjusted analysis. The likelihood of HgbA1c testing among patients with stage 2 to 3 CRC significantly increased from the phase before diagnosis to the surveillance phase, though this group lagged significantly behind controls in the phase after initial treatment. Although patients with stage 0 to $1 \mathrm{CRC}$ had a higher likelihood of lipid testing before diagnosis, by the phase after initial treatment the likelihood of lipid testing among patients with stage 0 to $1 \mathrm{CRC}$ fell to that of controls and remained at roughly the same level as controls throughout subsequent phases (Figure 2). The likelihood of lipid testing among patients with stage 2 to 3 CRC lagged behind controls in the phase after initial treatment and behind both controls and patients with stage 0 to $1 \mathrm{CRC}$ in the surveillance phase (Figure 2). Adjusting for physician visits did not change these findings (results not shown).

\section{Discussion}

Elderly patients with stage 2 to 3 CRC demonstrated significant gains in influenza vaccination after cancer diagnosis, with even more notable gains in mammography. These unexpected findings refuted our hypothesis that cancer's intensive treatment would supplant this care. Instead, it may be that patients with stage 2 to $3 \mathrm{CRC}$, who are some of the least likely to use these services before diagnosis, are now receiving regular care from cancer care physicians who provide and/or advise use of these preventive services and are complying with these recommendations. Alternately, or in addition, these patients' noncancer care physicians may more actively promote these services after cancer diagnosis. More frequent physician visits only partially explained the gains in influenza vaccination and mammography, however, suggesting that physician encouragement combined with patient attention to preventive care contributed to this change. These findings match those of research examining preventive testing among breast cancer survivors and controls, ${ }^{20}$ though this study is the first to follow preventive service use from before diagnosis through 7 years of survival.

Later-stage CRC patients did not demonstrate the same pattern in diabetes care. Despite frequent physician visits, patients with stage 2 to 3 CRC had less consistent increases and sometimes decreased diabetes testing compared with controls after initial 
treatment. Chronic disease care is more complex, and cancer care physicians may feel less comfortable both advising about and providing these services. Whether this lesser focus on diabetes care (and potentially other chronic conditions) has a deleterious effect is unknown, but studies demonstrating higher mortality among patients with comorbid conditions in the first years after diagnosis suggest the importance of optimal management of comorbid conditions. ${ }^{8,10}$

What strategies might improve care quality for cancer patients' chronic conditions like diabetes? Although cancer diagnosis and treatment may divert attention from other medical conditions, cancer patients are well connected with the medical care system. Early on, cancer patients receive care from cancer care physicians as well as primary care physicians and noncancer care specialists, who may have conducted screening or initial symptom evaluation; further out from diagnosis, cancer survivors primarily receive care from primary care physicians and noncancer care specialists. ${ }^{21}$ Building on these physician resources to improve the quality of cancer patients' care is key. One important strategy is the development of strong partnerships between patients; their primary care physicians, who are specialists in chronic disease management; and their cancer care physicians. With strong partnerships in place, cancer care physicians could promote the importance of continuing primary care visits, and primary care physicians could ensure that oncologists receive historic clinical and psychosocial information that facilitates optimal patient care. Studies examining these partnerships emphasize communication from oncologists to primary care physicians to improve cancer-related care but do not highlight the importance of a bidirectional relationship or of cancer patients' other health concerns. ${ }^{2-24}$ The Institute of Medicine endorses a coordinated primary and specialty care approach to addressing cancer patients' needs, and suggests development of a survivorship care plan to facilitate optimal care. ${ }^{14}$ Future research systematically examining the effectiveness of strategies that enhance communication between primary and cancer care providers about cancer-related and chronic condition care is needed.

Neither controls nor cases uniformly received guideline-recommended preventive or diabetes care. This finding illustrates that neither frequent patient visits nor strong physician partnerships alone will ensure optimal preventive or chronic disease care. Continued implementation, evaluation, and development of care improvement strategies, such as chronic disease management models, to care for individuals with competing medical priorities such as a cancer diagnosis is important. ${ }^{25}$

Notably, patients with stage 0 to 1 CRC used medical care differently than both patients with stage 2 to 3 and controls, even before diagnosis. These early-stage CRC patients had the highest comorbidity rates and the highest hospitalization and outpatient visit rates before diagnosis. Their high annual preventive service use rates before diagnosis could be related to greater contact with health professionals or greater health-seeking behavior, which could in turn help explain their earlystage cancer diagnosis.

The many cancer cases in the longitudinal SEER-Medicare database made this study possible, yet these data are limited. They cannot document true preventive service rates. Influenza vaccination is available in many settings, including pharmacies, where patients may pay cash rather than submit a Medicare claim, and during hospitalization, where vaccination is bundled with other charges. In addition, this study's annual mammography rates, which measure the combination of annual screening or diagnostic mammography, do not represent every two year screening mammography as was covered by the Medicare program during the study period. Despite these deviations from true population-based preventive service rates, comparing these services over time or between study groups is valid.

An additional limitation is that few evidencebased chronic disease care measures are available in Medicare claims. We chose diabetes care markers recommended by the American Diabetes Association that were likely to be captured reliably by claims. HgbA1c, which monitors blood glucose control, is widely used to assess quality of care but has not been associated with improvement in outcomes such as diabetes-related mortality. ${ }^{26}$ Claims data are also susceptible to secular coding changes. This is most obvious in the comorbidity index, which increased over time (eg, mean comorbidity index for 70 year olds in 1993 was 0.120 and in 1999 was 0.140). Also notable is that SEER data do not record cancer recurrence, although we censored an individual's observation years starting with the first year in which there was evidence of treat- 
ment for recurrence (ie, chemotherapy and radiation administration). Because of attrition from death, the populations in each phase differed. To explore the influence of these population differences, we repeated analyses including only patients with at least one observation year in the survival phase and found similar point estimates but widened confidence intervals. Lastly, the study data are limited by their ages. Behavioral Risk Factor Surveillance System data demonstrate increases in flu vaccination, mammography, and cholesterol testing among the general population between the mid-1990s and late 2000s. ${ }^{27}$ However, given that there have been no major changes in the organization of oncology or primary care services during this time period, it is likely that the relationships between cancer diagnosis and receipt of preventive and diabetes services found in this study persist.

\section{Conclusion}

Cancer diagnosis and treatment are consuming medical experiences. The intensity of medical visits and side effects of treatment can be overwhelming. Although cancer care must be of primary concern, many cancer patients have other medical conditions that influence survival and quality of life. The intensity of cancer treatment offers opportunities as well as risks related to the general medical care of cancer patients. Cancer diagnosis is associated with an increase in receipt of preventive services such as influenza vaccination and mammography. However, the likely narrow focus on cancer during many medical visits does not promote care for chronic medical conditions. Improvement of this care requires additional strategies, such as strengthening partnerships between cancer patients, primary care physicians, and cancer care physicians. Yet these strategies alone are unlikely to bring chronic disease care to recommended levels because the relatively low annual rates of diabetes testing among controls show that effective strategies that improve care for all patients with chronic conditions such as diabetes are needed.

The authors would like to thank Barbara Matthews, for her assistance in developing the database used in this research; Denise Lishner, for carefully documenting the work of the research team; and Roger A. Rosenblatt, MD, MPH, MFR, and Joan Warren, RN, PhD, for their critical review of this manuscript.

\section{References}

1. Deimling GT, Sterns S, Bowman KF, Kahana B. The health of older-adult, long-term cancer survivors. Cancer Nurs 2005;28:415-24.

2. Ko C, Chaudhry S. The need for a multidisciplinary approach to cancer care. J Surg Res 2002;105:53-7.

3. Ogle KS, Swanson GM, Woods N, Azzouz F. Cancer and comorbidity: redefining chronic diseases. Cancer 2000;88:653-63.

4. Seo PH, Pieper CF, Cohen HJ. Effects of cancer history and comorbid conditions on mortality and healthcare use among older cancer survivors. Cancer 2004;101:2276-84.

5. Bach PB, Schrag D, Brawley OW, Galaznik A, Yakren S, Begg CB. Survival of blacks and whites after a cancer diagnosis. JAMA 2002;287:2106-13.

6. Earle CC. Influenza vaccination in elderly patients with advanced colorectal cancer. J Clin Oncol 2003; 21:1161-6.

7. Meyerhardt JA, Catalano PJ, Haller DG, et al. Impact of diabetes mellitus on outcomes in patients with colon cancer. J Clin Oncol 2003;21:433-40.

8. Satariano WA, Ragland DR. The effect of comorbidity on 3-year survival of women with primary breast cancer. Ann Intern Med 1994;120:104-10.

9. Tammemagi CM, Nerenz D, Neslund-Dudas C, Feldkamp C, Nathanson D. Comorbidity and survival disparities among black and white patients with breast cancer. JAMA 2005;294:1765-72.

10. West DW, Satariano WA, Ragland DR, Hiatt RA. Comorbidity and breast cancer survival: a comparison between black and white women. Ann Epidemiol 1996;6:413-9.

11. Ramsey SD, Berry K, Moinpour C, Giedzinska A, Andersen MR. Quality of life in long term survivors of colorectal cancer. Am J Gastroenterol 2002;97: 1228-34.

12. Earle CC, Neville BA. Under use of necessary care among cancer survivors. Cancer 2004;101:1712-9.

13. Snyder CF, Earle CC, Herbert RJ, Neville BA, Blackford AL, Frick KD. Preventive care for colorectal cancer survivors: a 5 -year longitudinal study. J Clin Oncol 2008;26:1073-9.

14. Hewitt M, Greenfield S, Stovall E, eds. From cancer patient to cancer survivor: lost in transition. Washington, DC: National Academies Press; 2005.

15. National Cancer Institute. SEER-Medicare: about the data files. Available at: http://healthservices. cancer.gov/seermedicare/aboutdata/. Accessed August $18,2010$.

16. Update on adult immunization. Recommendations of the Immunization Practices Advisory Committee (ACIP). MMWR Recomm Rep 1991;40:1-94.

17. U.S. Preventive Services Task Force. Guide to clinical preventive services, second edition. Rockville, MD: U.S. Department of Health and Human Services; 1996. 
18. Klabunde CN, Legler JM, Warren JL, Baldwin LM, Schrag D. A refined comorbidity measurement algorithm for claims-based studies of breast, prostate, colorectal, and lung cancer patients. Ann Epidemiol 2007;17:584-90.

19. Zhang J, Yu KF. What's the relative risk? A method of correcting the odds ratio in cohort studies of common outcomes. JAMA 1998;280:1690-1.

20. Earle CC, Burstein HJ, Winer EP, Weeks JC. Quality of non-breast cancer health maintenance among elderly breast cancer survivors. J Clin Oncol 2003; 21:1447-51.

21. Pollack LA, Adamache W, Ryerson AB, Eheman CR, Richardson LC. Care of long-term cancer survivors: physicians seen by Medicare enrollees surviving longer than 5 years. Cancer 2009;115:5284-95.

22. Johansson B, Berglund G, Hoffman K, Glimelius B, Sjoden PO. The role of the general practitioner in cancer care and the effect of an extended information routine. Scand J Prim Health Care 2000;18:143-8.

23. Kousgaard KR, Nielsen JD, Olesen F, Jensen AB. General practitioner assessment of structured oncological information accompanying newly referred cancer patients. Scand J Prim Health Care 2003;21: $110-4$.

24. Nielsen JD, Palshof T, Mainz J, Jensen AB, Olesen F. Randomised controlled trial of a shared care programme for newly referred cancer patients: bridging the gap between general practice and hospital. Qual Saf Health Care 2003;12:263-72.

25. Bodenheimer $T$. Interventions to improve chronic illness care: evaluating their effectiveness. Dis Manag 2003;6:63-71.

26. Intensive blood-glucose control with sulphonylureas or insulin compared with conventional treatment and risk of complications in patients with type 2 diabetes (UKPDS 33). UK Prospective Diabetes Study (UKPDS) Group. Lancet 1998;352:837-53.

27. Centers for Disease Control. Prevalence and trends data. Available at: http://apps.nccd.cdc.gov/BRFSS/. Accessed August 18, 2010.

\section{Appendix: Medicare Claim Codes for Study Variables}




\begin{tabular}{|c|c|c|}
\hline Variables & Claim Type & Medicare Claim Codes \\
\hline Mammography & HCPCS/CPT & 76090, 76091, 76092 \\
\hline \multirow[t]{3}{*}{ Influenza vaccination } & HCPCS/CPT & 90724, G0008, 90657-90660, Q0124 \\
\hline & ICD-9-CM & V048, V066 \\
\hline & ICD-9-P & 99.52 \\
\hline HgbA1c & HCPCS/CPT & 83036 \\
\hline Lipid testing & HCPCS/CPT & $\begin{array}{l}80061,80062,82465,82470,83700,83705,83715- \\
\quad 83721,84478\end{array}$ \\
\hline \multirow[t]{3}{*}{ Evidence of metastatic disease } & HCPCS/CPT & $\begin{array}{l}36246,36247,47120,47122,47125,47130,47370, \\
\quad 47371,47380,47381,47382,76362,76394,76490 \\
\quad 36260,47100, \mathrm{C} 2618\end{array}$ \\
\hline & ICD-9-CM & $\begin{array}{l}\text { 197.0, 197.00, 197.04, 197.08, 197.1, 197.2, 197.3 } \\
\text { 197.7, 197.70, 197.8, 198.3, 198.4, 198.41, 198.45, } \\
\text { 198.48, 198.5, 198.51 }\end{array}$ \\
\hline & ICD-9-P & $\begin{array}{l}50.2,50.20,50.21,50.22,50.29,50.3,50.30,50.4 \\
\quad 50.40\end{array}$ \\
\hline \multicolumn{3}{|l|}{ Recurrence treatment } \\
\hline \multirow[t]{3}{*}{ Chemotherapy: } & HCPCS/CPT & $\begin{array}{l}\text { 96408, 96410, 96412, 96414, 96520, 96530, 96545 } \\
\text { 96549, J9190, J0640, J9200, Q0083, Q0084, } \\
\text { Q0085 }\end{array}$ \\
\hline & ICD-9-CM & V58.1, E933.1, V66.2, V67.2 \\
\hline & ICD-9-P & 99.25 \\
\hline \multirow[t]{4}{*}{ Radiation therapy: } & HCPCS/CPT & $\begin{array}{l}\text { 77261-63, 77280, 77285, 77290, 77295, 77299, } \\
\text { 77300, 77305, 77310, 77315, 77321, 77326-8, } \\
\text { 77331-4, 77336, 77370, 77399, 77401-17, 77419- } \\
\text { 32, 77470, 77490, 77499, 77750, 77761-3, 77776- } \\
\text { 8, 77781-4, 77789-90, 77797, 77799, }\end{array}$ \\
\hline & ICD-9-CM & V66.1, V67.1, V58.0 \\
\hline & ICD-9-P & $92.20-92.29$ \\
\hline & Revenue center & 0333 \\
\hline
\end{tabular}

HCPCS, Healthcare Common Procedure Coding System; CPT, Current Procedural Terminology; ICD-9-CM, International Classification of Diseases, $9^{\text {th }}$ Revision, Clinical Modification; ICD-9-P, ICD-9-CM Procedure. 